\title{
Revealing gene clusters associated with the development of cholangiocarcinoma, based on a time series analysis
}

\author{
JIANYU WU $^{1}$, ZHIFU XIAO $^{1}$, XIULEI ZHAO ${ }^{2}$ and XIANGSONG WU ${ }^{1}$ \\ ${ }^{1}$ Department of General Surgery, First Affiliated Hospital of Harbin Medical University, Harbin, Heilongjiang 150001; \\ ${ }^{2}$ Department of General Surgery, The Central Hospital of Cangzhou, Cangzhou, Hebei 061000, P.R. China
}

Received December 17, 2013; Accepted June 26, 2014

DOI: $10.3892 / \mathrm{mmr} .2015 .3216$

\begin{abstract}
Cholangiocarcinoma (CC) is a rapidly lethal malignancy and currently is considered to be incurable. Biomarkers related to the development of $\mathrm{CC}$ remain unclear. The present study aimed to identify differentially expressed genes (DEGs) between normal tissue and intrahepatic $\mathrm{CC}$, as well as specific gene expression patterns that changed together with the development of CC. By using a two-way analysis of variance test, the biomarkers that could distinguish between normal tissue and intrahepatic CC dissected from different days were identified. A $\mathrm{k}$-means cluster method was used to identify gene clusters associated with the development of CC according to their changing expression pattern. Functional enrichment analysis was used to infer the function of each of the gene sets. A time series analysis was constructed to reveal gene signatures that were associated with the development of $\mathrm{CC}$ based on gene expression profile changes. Genes related to $\mathrm{CC}$ were shown to be involved in 'mitochondrion' and 'focal adhesion'. Three interesting gene groups were identified by the k-means cluster method. Gene clusters with a unique expression pattern are related with the development of $\mathrm{CC}$. The data of this study will facilitate novel discoveries regarding the genetic study of $\mathrm{CC}$ by further work.
\end{abstract}

\section{Introduction}

Cholangiocarcinoma (CC) is an adenocarcinoma that forms glands or secretes significant amounts of mucins. These are composed of mutated epithelial cells that originate in the bile ducts, which drain bile from the liver into the small intestine (1-3). CC is currently considered to be an incurable and rapidly lethal malignancy. Currently, surgery has been shown to be the only existing curative treatment for CC $(4,5)$. Even though the incidence rate of $\mathrm{CC}$ is considerably low, this rate has been rising over the past decades (6-8).

Correspondence to: Dr Xiangsong Wu, Department of General Surgery, First Affiliated Hospital of Harbin Medical University, 23 Youzheng Street, Harbin, Heilongjiang 150001, P.R. China E-mail: 13633621389@163.com

Key words: cholangiocarcinoma, $k$-means cluster, association study
Both environmental and genetic factors can affect the risk of CC. One of the most common factors that cause $\mathrm{CC}$ in the western world is primary sclerosing cholangitis (PSC), which is an inflammatory disease closely related with ulcerative colitis. It was suggested that PSC can increase the risk of CC by $\sim 10-15 \%$ (9). However, the molecular mechanism of PSC increasing the risk of CC remains largely unknown $(10,11)$. Some liver diseases caused by parasitic infection have been considered causative of CC. Previous research has identified that colonization with the liver flukes Opisthorchis viverrini and Clonorchis sinensis is associated with the development of CC (12-14). Numerous studies have suggested that chronic liver disease can also increase the risk of CC, including viral hepatitis related liver disease and alcoholic liver disease $(15,16)$. To date, numerous genetic factors have also been investigated to show evidences of being involved in the outcome of CC, including p53, cyclins, mucins and CRP (17). Aneuploidy has additionally been suggested to have a significant role in the development of CC.

Despite previous research aiming to reveal the genetic association with the risk of $\mathrm{CC}$, few studies have focused on gene expression changes together with the development of $\mathrm{CC}$ over different days. The present study aimed to generate a time series analysis of $\mathrm{CC}$ at the gene expression level in order to identify novel gene patterns that are associated with the development of CC (18).

\section{Materials and methods}

Microarray data for $C C$. The study was approved by the ethics committee of Haerbin Medical University. A gene expression profile dataset related to $\mathrm{CC}$ development formed over different days was obtained from the Gene Expression Omnibus (GEO, http://www.ncbi.nlm.nih.gov/geo/) (19) in the National Center for Biotechnology Information (NCBI), with accession number GSE15498. There were 21 samples in total, including nine normal tissues, nine intrahepatic CC tissues and three metastasis tissues. All microarray data were generated on the platform of the Affymetrix Rat Genome 2302.0 Array (Affymetrix, Inc., Santa Clara, CA, USA).

Microarray data pre-processing. The original CEL files were downloaded from NCBI and the standard array processing procedures of the Affymetrix Rat Genome 2302.0 Array 
were followed. The 'affy' package in R (20) was used to extract signal values, which were then scaled to a mean of 100 , followed by $\log 2$ transformation of the array signals. A quantile normalization method (21) was used to normalize the expression value across samples. Finally, all probe set IDs were transformed to gene symbol IDs based on the documentation of the manufacturer for downstream analysis.

Principle component analysis (PCA) and pair-wise correlation analysis. The R (http://www.r-project.org/) 'princomp' function in the package 'stats', was used to perform the (PCA) based on the gene expression level following normalization across all 21 samples. Furthermore, a pair-wise correlation matrix was calculated among these samples. A heatmap based on the correlation matrix was constructed, as well as hierarchical clustering, by using 'euclidean' distance as the input.

Differentially expressed genes (DEGs) analysis. To detect the DEGs among normal, CC and metastasis tissue, a two-way analysis of variance (ANOVA) test was performed (22) with the gene expression profile as an input. Genes with a $\mathrm{P}<0.0001$ were considered to be associated with the development of CC. To investigate whether these DEGs were significant, a permutation test to evaluate the false discovery rate (FDR) was additionally performed.

Functional enrichment analysis. The Database for Annotation, Visualization and Integrated Discovery (DAVID) (23) was used to analyze the enriched Gene Ontology (GO) and Kyoto Encyclopedia of Genes and Genomes (KEGG) pathway terms (24). All expressed genes were treated as the background for this test.

$k$-means cluster analysis. To perform a comprehensive analysis of genes associated with CC genesis and identify gene clusters that may function in the development of $\mathrm{CC}$, a k-means cluster method (25) was used to classify genes into different groups according to their change in expression pattern. Specifically, the mean expression value for each differentially expressed gene within each group (normal and tumor tissues from 10 , 15 and 25 days, respectively, as well as metastasis tissue) was calculated. Next, a z-score transformation for the gene expression profile with mean values across sample groups described above based on a standard normalization method was performed in order to eliminate the absolute expression value effect and focus on the pattern change. Finally, a k-means cluster method was carried out for these significantly DEGs, according to their $\mathrm{z}$-transformed expression profile $(\mathrm{k}=8)$.

Gene module functional analysis. For each gene module identified by the k-means cluster method, DAVID was used to analyze the enriched GO terms, as well as KEGG pathway terms. Functional terms with $\mathrm{P}<0.05$ were considered to indicate statistically significant enriched functions.

\section{Results}

Genes associated with $C C$. There were 21 samples used in total in the present study, which were grouped into three types, including, 12 intrahepatic CC samples formed at different days ranging from 10 to 25 days, 12 normal control samples taken from the same days as a pair-wise comparison with intrahepatic CC samples and three metastasis samples used as a positive control. A PCA was conducted to demonstrate the association between samples in terms of their gene expression profile. The results showed a clear separation pattern between control samples and samples with disease (Fig. 1A). Hierarchical cluster results based on the pair-wise correlation matrix of each sample (shown in Fig. 1B) also suggest that control samples and disease samples may be successfully separated in terms of their gene expression profile. A two-way ANOVA was used to detect genes differentially expressed among these three groups of samples, as well as between the samples that derived from different days. Genes with $\mathrm{P}<0.0001$ were defined as CC related genes. In total, 4,136 genes were identified that showed significantly different levels of expression between at least two types of samples. To evaluate whether these genes were significant rather than random, a permutation test was performed by shuffling the samples and repeating the ANOVA test 100,000 times. By using $\mathrm{P}<0.0001$ as a cutoff, an $\mathrm{FDR}=0.03 \%$ was produced.

Functional analysis of CC gene signatures. In order to fully determine the association between the DEGs and the phenotype of $\mathrm{CC}$, a hypergeometric test to detect the enriched GO terms was used. DAVID was used to perform this analysis. Only over-represented GO terms with $\mathrm{P}<0.05$ following Bonferroni correction were considered to be significantly enriched GO terms. The top ten enriched GO terms are listed in Table I. The enriched terms, such as intracellular organelle lumen, membrane-enclosed lumen, organelle lumen have been associated with CC in several previous studies $(26,27)$.

Pathway analysis of CC gene signatures. To further understand the association between the DEGs and their involvement in the development of CC, the type of molecular pathways that were significantly enriched were tested using the KEGG definition. KEGG terms with a $\mathrm{P}<0.05$ following Bonferroni correction were selected (Table II). As expected, a significantly enriched pathway termed 'mitochondrion', was identified, which is directly linked with CC (28), suggesting that these DEGs participate in the development of CC.

Identification of gene clusters associated with specific CC pathways. For those genes that were differentially expressed between normal and tumor tissues at various developmental stages, it was asked what was their changing pattern as well as each pattern's biological function, in terms of their association with CC. A k-means cluster method was used to classify the genes into different modules according to their change in expression pattern. To eliminate the absolute expression level effect and only focus on the gene expression change curve difference, the gene expression profile was normalized based on the z-score transformation. Eight patterns were produced in total (Fig. 2). Genes that show a consistent changing pattern along with developmental stages of $\mathrm{CC}$ (such as from 10 to15 days and to 25 days) should be considered to be associated with the chronological development of CC, since it was hypothesized that a good correlation between change in gene expression and change in disease phenotype is strong evidence of association. 
Table I. Top ten enriched GO terms.

\begin{tabular}{llccc}
\hline GO ID & \multicolumn{1}{c}{ Term } & Count & Bonferroni & Fold enrichment \\
\hline GO:0005829 & Cytosol & 384 & $2.69 \times 10^{-23}$ & 3.412796 \\
GO:0005739 & Mitochondrion & 409 & $2.37 \times 10^{-20}$ & 3.078936 \\
GO:0031974 & Membrane-enclosed lumen & 405 & $7.46 \times 10^{-19}$ & 2.782038 \\
GO:0043233 & Organelle lumen & 394 & $3.12 \times 10^{-18}$ & 2.423745 \\
GO:0070013 & Intracellular organelle lumen & 380 & $1.98 \times 10^{-17}$ & 3.017707 \\
GO:0006414 & Translational elongation & 61 & $3.36 \times 10^{-16}$ & 2.548286 \\
GO:0022627 & Cytosolic small ribosomal subunit & 31 & $1.10 \times 10^{-11}$ & 1.543415 \\
GO:0044429 & Mitochondrial part & 185 & $1.21 \times 10^{-11}$ & 2.26445 \\
GO:0044445 & Cytosolic part & 60 & $4.53 \times 10^{-11}$ & $4.91 \times 10^{-11}$
\end{tabular}

GO, gene ontology.

Table II. Enriched Kyoto Encyclopedia of Genes and Genomes pathways.

\begin{tabular}{llccc}
\hline KEGG ID & \multicolumn{1}{c}{ Term } & Count & Bonferroni & Fold enrichment \\
\hline rno03010 & Ribosome & 64 & $6.62 \times 10^{-23}$ & 3.412796 \\
rno00280 & Valine, leucine and isoleucine degradation & 32 & $1.83 \times 10^{-08}$ & 3.078936 \\
rno03030 & DNA replication & 22 & $3.75 \times 10^{-04}$ & 2.782038 \\
rno00071 & Fatty acid metabolism & 23 & 0.004411 & 2.423745 \\
rno00410 & P-alanine metabolism & 15 & 0.008072 & 3.017707 \\
rno00640 & Propanoate metabolism & 19 & 0.012183 & 2.548286 \\
rno04510 & Focal adhesion & 68 & 0.016978 & 1.543415 \\
rno00380 & Tryptophan metabolism & 22 & 0.025275 & 2.26445 \\
rno04810 & Regulation of actin cytoskeleton & 70 & 0.049876 & 1.482382 \\
\hline
\end{tabular}

KEGG, Kyoto Encyclopedia of Genes and Genomes.

\section{A}

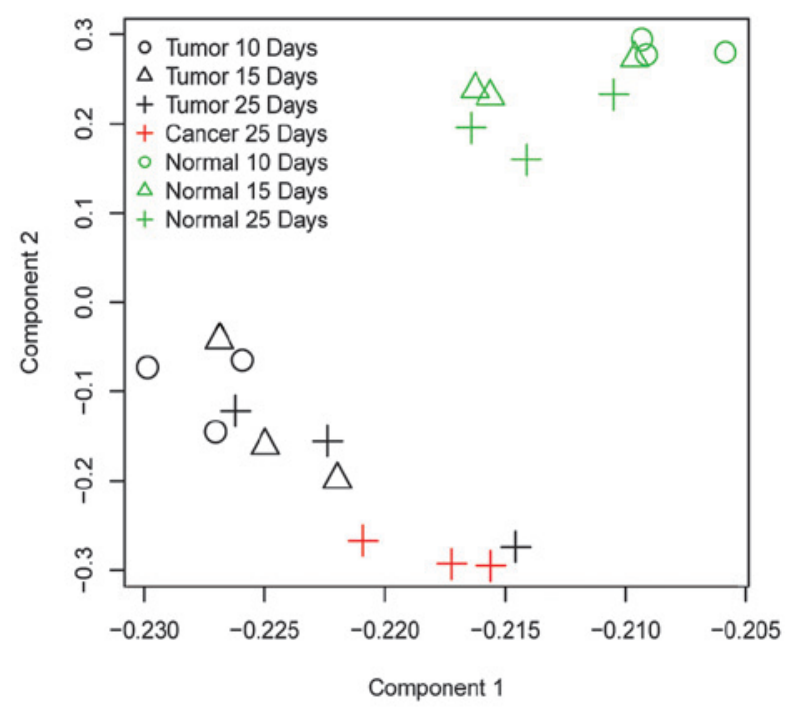

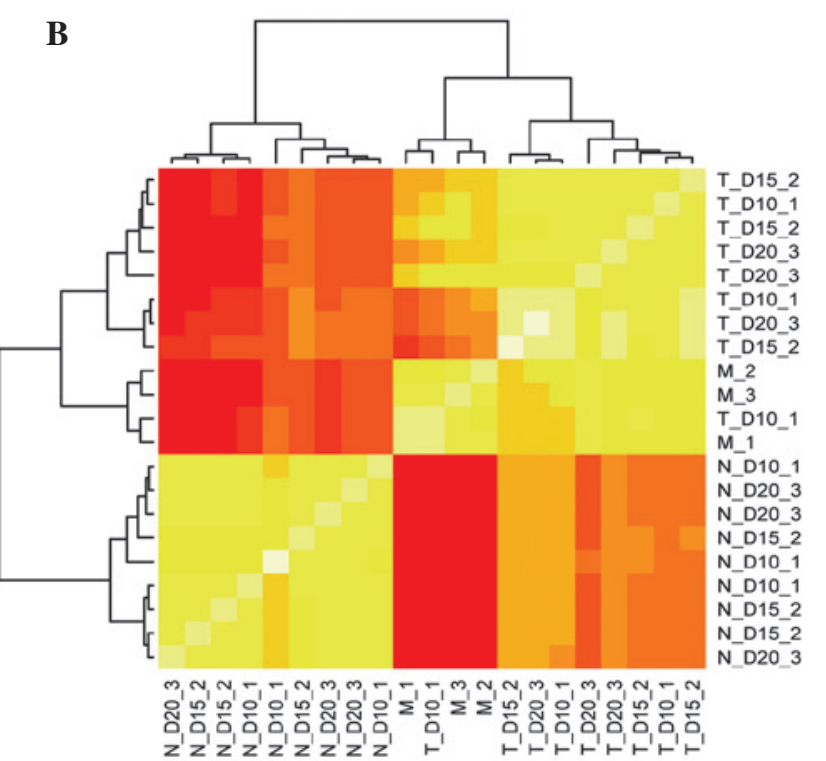

Figure 1. Principle component analysis and pair-wise correlation cluster analysis of 21 samples. (A) The principle component analysis of 21 samples. Black represents tumor samples; green represents normal samples; red represents metastasis samples. The different symbols represent the different histological days of each sample (as shown in the figure). (B) A heatmap of 21 samples showing the hierarchical clustering of all 21 samples based on the pair-wise correlation matrix. T, tumor; N, normal; M, metastasis; D, days. 
A

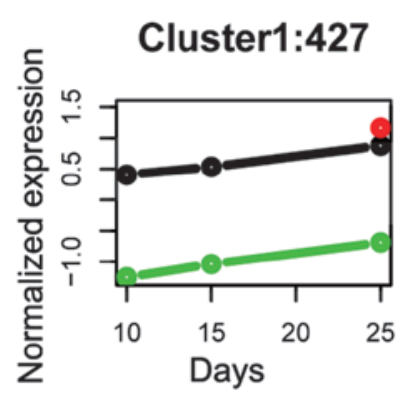

D

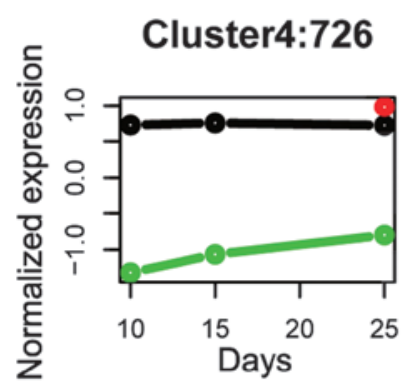

G

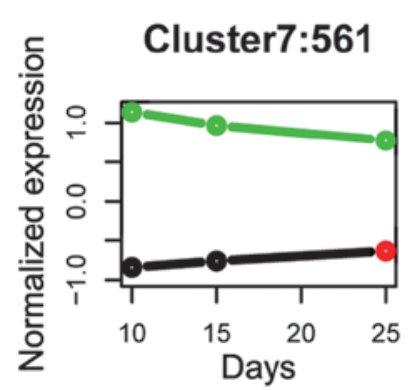

B

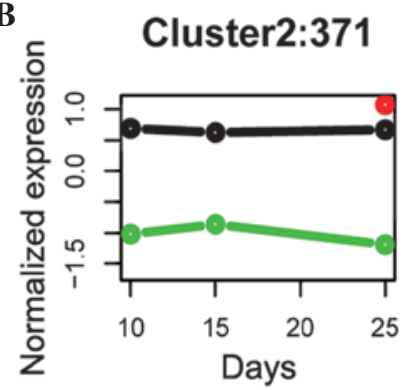

$\mathbf{E}$

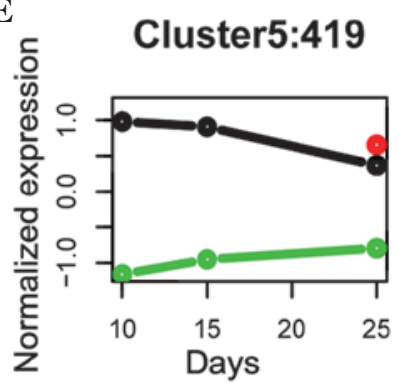

$\mathbf{H}$

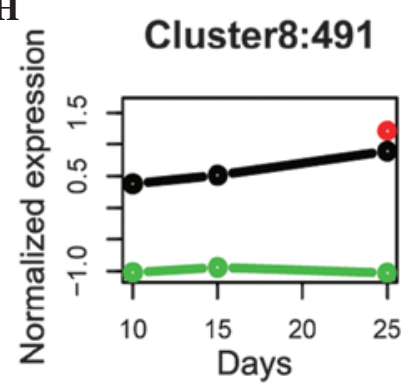

C

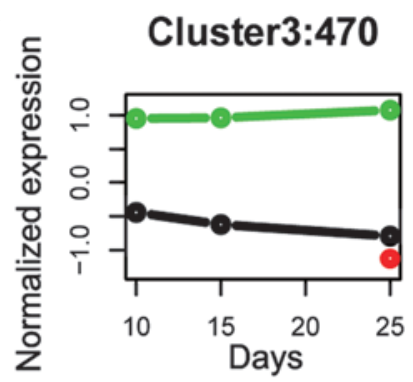

$\mathbf{F}$

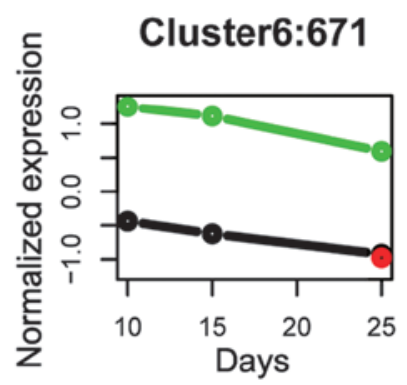

Figure 2. (A-H) Clusters of all significant differentially-expressed genes based on the $k$-mean cluster method. The X-axis represents the histological days of each sample and the Y-axis represents the z-transformed gene expression level. Black represents tumor samples; green represents normal samples; Red represents metastasis samples. The number above each figure represents the number of genes within each cluster.

Genes that show a pattern difference between tumor and normal samples across the same chronological days should also be firstly considered to have functional impact, especially for those genes that have an opposite pattern. Notably, three interesting gene clusters were identified: i) 470 downregulated genes along with the developmental stage of $\mathrm{CC}$ while the control curve was flat (Fig. 2C); ii) 419 downregulated genes along with the developmental stage of $\mathrm{CC}$ while the control curve showed a slight upregulation (Fig. 2E); iii) 491 upregulated genes along with the developmental stage of $\mathrm{CC}$ while the control curve was flat (Fig. 2H). These genes were possible candidate biomarkers of $\mathrm{CC}$ in specific pathways, which may function in the development of $\mathrm{CC}$ and should be studied further in future studies.

To investigate the functional importance of the gene clusters mentioned, the enriched function of all three interested gene groups was investigated. For gene cluster-3 (Fig. 2C), it was identified that genes were enriched in cell structural related functions such as 'structural molecule activity', 'microtubule', and 'microtubule skeleton'. Genes in cluster-5 (Fig. 2E) were predominantly enriched in gene transcription and translation related pathways, suggesting that these genes may participate in facilitating translation of important genes that are associated with CC. Notably, genes in module-5 were found to be additionally enriched in a KEGG pathway termed 'mitochondrion', which was suggested to be a direct evidence that these genes were related with the development of CC (28). This indicated that genes downregulated with the development stage of CC were candidate biomarkers of CC. Genes in cluster-8 (Fig. 2H) were enriched in the GO terms 'Focal adhesion' and 'Regulation of actin cytoskeleton' $(\mathrm{P}<0.05)$.

\section{Discussion}

$\mathrm{CC}$ is considered to be an incurable cancer, as well as a rapid lethal malignancy, unless the primary tumor and any metastases can be fully removed surgically. To date, only a few genetic regions have been investigated to show an association with the development of $\mathrm{CC}$, including p53, cyclins, mucins, and CRP (17). In the present study, a gene expression profile change along with the chronological development of $\mathrm{CC}$, was performed in a time-series dataset of CC patient samples by 
using the same chronological samples as a control. The PCA revealed that normal samples may be obviously distinguished both from tumor and cancer samples (Fig. 1A), demonstrating that the most marked change in the gene expression level is between normal developed tissue and tumor tissue. It was additionally noticed, however, that there was a fundamental difference between tumor tissues with various developmental stages, ranging from 10 to 25 days. Notably, there was a consistent change in gene expression pattern as indicated by the PCA, in particular in the late developmental stages of tumor tissue (25 days) is closer to the stage of cancerous tissue (Fig. 1A). A heatmap based on pair-wise correlation of each sample in terms of their gene expression profile, generated the same conclusion as the PCA (Fig. 1B). These data suggested that the development of CC from early to late stage was generally a consistent process at the molecular level, reflecting that the transcriptome status is a potential molecular symbol of the developmental stage of CC. It is hypothesized that this result will facilitate the molecular diagnostic research of $\mathrm{CC}$ in the future.

A two-way ANOVA was performed in order to identify candidate gene markers that were differentially expressed between normal and tumor tissues, with various developmental stages. In total, 4,136 DEGs, of which most were significantly differentially expressed between normal and intrahepatic CC tissues $(\mathrm{P}<0.0001)$, we identified. Permutation testing provided a considerably low FDR (0.03\%), suggesting these DEGs were truly significant molecular markers rather than background noise. Of these DEGs, numerous genes have been previously identified to be CC-associated genes, including GAPDH, CITED4 and CLDN4 (17,30,31). Notably, a well studied zinc finger gene, SNAI1, which is considered to be associated in the molecular pathways of CC development was identified in the DEG gene list (4.1 fold change; $\mathrm{P}=1.08 \mathrm{e}-07)(31-33)$. SNAI1 was shown to be upregulated in intrahepatic CC, which is consistent with the observations of the present study. The function of SNAI1 is to suppress the expression of E-cadherin that mediates cell-to-cell adhesion in order to regulate tumor progression and metastases $(26,35)$.

GO enrichment analysis revealed that the significant DEGs were likely to be involved in functions such as intracellular organelle lumen, membrane-enclosed lumen and organelle lumen. These terms were significantly associated with the development of $\mathrm{CC}$ as additionally shown in previous research $(26,36)$. This suggested that our analysis was robust and consistent with previous studies. In addition, mitochondrial functions (including mitochondrion, mitochondrial part) were also significantly enriched (Bonferroni $\mathrm{P}<1 \mathrm{e}-12$ ), suggesting that mitochondrion-related molecular pathways were associated with the development of CC. In agreement with this, previous studies have shown that mitochondrion-mediated specific cell type apoptosis is an important cause for the development of metastases in human CC cells. Okaro et al (28) showed that a mitochondrial benzodiazepine receptor antagonist, $\mathrm{Pk} 11195$, can downregulate the apoptosis tolerance in human CC cells (28). Another study found that Mcl-1 can mediate apoptosis-inducing ligand resistance in human $\mathrm{CC}$ cells (37). Taken together, these data suggest that other genes in the gene list produced in the present study, that are associated with mitochondrion function, are potential candidate genes that should be studied in the future in the context of CC. KEGG pathway enrichment analysis provided additional information regarding the linkage between molecular pathways and the phenotype of CC (Table II). It was observed that significantly enriched terms, such as 'Focal adhesion' and 'Regulation of actin cytoskeleton' $(\mathrm{P}<0.05)$ were well studied pathways that have been previously associated with metastasis $(38,39)$, thus adding to the reliability of these DEGs. In conclusion, it is considered that these DEGs detected by two-way ANOVA represent true molecular markers that can distinguish between normal intrahepatic duct cells and intrahepatic CC cells.

To further investigate genes that have different functions in the tumorigenesis and development of CC, a k-means cluster method was performed for these DEGs, in order to identify gene markers that could distinguish each other by gene expression pattern. Specifically, genes that showed a consistent change with developmental stages (from 10 to 25 days) and a different change in expression curve in that we believe a correlation between gene expression and phenotypic change of CC is always a strong evidence of association were investigated. Distinguishable patterns between tumor and normal samples should be considered to be of importance in terms of gene function in specific pathways of CC. Based on these two criteria, $3 / 8$ clusters produced notable patterns of expression. In cluster-3, genes in normal tissues were highly expressed as compared with tumor tissues, and were expressed in a steady state across different days. In comparison the genes in the tumor tissues were downregulated together with the development of CC. Enrichment tests suggested that genes within this cluster were involved in pathways including 'structural molecule activity', 'microtubule' and 'microtubule skeleton', which have been linked with tumorigenesis in several previous studies $(40,41)$. Genes in cluster-5 were also shown to have a downregulation expression pattern in the developmental stages of CC. However, genes in this group were highly expressed in tumor tissues as compared with normal tissues. Pathway enrichment analysis showed that these genes were enriched in mitochondrion related pathways, suggesting they may function in cell apoptosis-induced pathways. Interestingly, SNAI1 was consistently within this cluster, indicating that studying other genes within this cluster may provide a link between SNAI1 regulation in the development of $\mathrm{CC}$. For cluster-8, genes that were upregulated across tumor samples whilst maintaining flat expression across normal samples were identified. GO enrichment analysis additionally suggested that genes within this group were related with 'Focal adhesion' and 'Regulation of actin cytoskeleton', raising the possibility that these genes may be tumorigenesis related markers associated with $\mathrm{CC}$ development.

In conclusion, the present study has conducted a time series analysis to reveal gene signatures that are associated with the development of $\mathrm{CC}$, based on changes in the gene expression profile. Genes related to $\mathrm{CC}$ were shown to be involved in cancer pathways such as 'mitochondrion' and 'focal adhesion'. Three interesting gene groups were identified by a k-means cluster method based on a normalized gene expression profile, of which each showed enrichment functions associated with the development of CC. It is considered that this work is of great importance and will lead to novel discoveries regarding the genetic study of $\mathrm{CC}$. 


\section{References}

1. Tsao JI, Nimura Y, Kamiya J, et al: Management of hilar cholangiocarcinoma: comparison of an American and a Japanese experience. Ann Surg 232: 166-174, 2000.

2. Nakeeb A, Pitt HA, Sohn TA, et al: Cholangiocarcinoma. A spectrum of intrahepatic, perihilar, and distal tumors. Ann Surg 224: 463-473.

3. Rajagopalan V, Daines WP, Grossbard ML and Kozuch P: Gallbladder and biliary tract carcinoma: A comprehensive update, Part 1. Oncology (Williston Park) 18: 889-896, 2004.

4. Weber SM, DeMatteo RP, Fong Y, Blumgart LH and Jarnagin WR: Staging laparoscopy in patients with extrahepatic biliary carcinoma. Analysis of 100 patients. Ann Surg 235: 392-399, 2002.

5. Callery MP, Strasberg SM, Doherty GM, Soper NJ and Norton JA: Staging laparoscopy with laparoscopic ultrasonography: optimizing resectability in hepatobiliary and pancreatic malignancy. J Am Coll Surg 185: 33-39, 1997.

6. Miyakawa S, Ishihara S, Horiguchi A, et al: Biliary tract cancer treatment: 5,584 results from the Biliary Tract Cancer Statistics Registry from 1998 to 2004 in Japan. J Hepatobiliary Pancreat Surg 16: 1-7, 2009.

7. Landis SH, Murray T, Bolden S and Wingo PA: Cancer statistics, 1998. CA Cancer J Clin 48: 6-29, 1998.

8. Patel T: Worldwide trends in mortality from biliary tract malignancies. BMC Cancer 2: 10, 2002.

9. Burak K, Angulo P, Pasha TM, et al: Incidence and risk factors for cholangiocarcinoma in primary sclerosing cholangitis. Am J Gastroenterol 99: 523-526, 2004

10. Khorsandi SE, Salvans S, Zen Y, et al: Cholangiocarcinoma complicating recurrent primary sclerosing cholangitis after liver transplantation. Transpl Int 24: e93-e96, 2011.

11. Kaya M, de Groen PC, Angulo P, et al: Treatment of cholangiocarcinoma complicating primary sclerosing cholangitis: the Mayo Clinic experience. Am J Gastroenterol 96: 1164-1169, 2001.

12. Chaiyarit P, Sithithaworn P, Thuwajit $C$ and Yongvanit $P$ : Detection of salivary antibodies to crude antigens of Opisthorchis viverrini in opisthorchiasis and cholangiocarcinoma patients. Clin Oral Investig 15: 477-483, 2011.

13. Hughes NR, Pairojkul C, Royce SG, Clouston A and Bhathal PS: Liver fluke-associated and sporadic cholangiocarcinoma: an immunohistochemical study of bile duct, peribiliary gland and tumour cell phenotypes. J Clin Pathol 59: 1073-1078, 2006

14. Shin HR, Lee CU, Park HJ, et al: Hepatitis B and C virus, Clonorchis sinensis for the risk of liver cancer: a case-control study in Pusan, Korea. Int J Epidemiol 25: 933-940, 1996.

15. Shaib YH, El-Serag HB, Davila JA, Morgan R and McGlynn KA: Risk factors of intrahepatic cholangiocarcinoma in the United States: a case-control study. Gastroenterology 128: 620-626, 2005

16. Sorensen HT, Friis S, Olsen JH, et al: Risk of liver and other types of cancer in patients with cirrhosis: a nationwide cohort study in Denmark. Hepatology 28: 921-925, 1998.

17. Briggs CD, Neal CP, Mann CD, et al: Prognostic molecular markers in cholangiocarcinoma: a systematic review. Eur J Cancer 45: 33-47, 2009.

18. Storto PD, Saidman SL, Demetris AJ, et al: Chromosomal breakpoints in cholangiocarcinoma cell lines. Genes Chromosomes Cancer 2: 300-310, 1990.

19. Barrett T and Edgar R: Gene expression omnibus: microarray data storage, submission, retrieval, and analysis. Methods Enzymol 411: 352-369, 2006.

20. Gautier L, Cope L, Bolstad BM and Irizarry RA: affy - analysis of Affymetrix GeneChip data at the probe level. Bioinformatics 20: 307-315, 2004.
21. Hu J and He X: Enhanced quantile normalization of microarray data to reduce loss of information in gene expression profiles. Biometrics 63: 50-59, 2007.

22. Perneger T and Perrier A: Analysis of variance, part 1 (the t-test and ANOVA). Rev Mal Respir 21: 797-801, 2004 (In French).

23. Huang da W, Sherman BT, Tan Q, et al: DAVID Bioinformatics Resources: expanded annotation database and novel algorithms to better extract biology from large gene lists. Nucleic Acids Res 35: W169-W175, 2007.

24. Kanehisa M and Goto S: KEGG: kyoto encyclopedia of genes and genomes. Nucleic Acids Res 28: 27-30, 2000.

25. Beauchaine TP and Beauchaine RJ 3rd: A comparison of maximum covariance and $\mathrm{K}$-means cluster analysis in classifying cases into known taxon groups. Psychol Methods 7: 245-261, 2002.

26. Sirica AE: The role of cancer-associated myofibroblasts in intrahepatic cholangiocarcinoma. Nat Rev Gastroenterol Hepatol 9: 44-54, 2011.

27. Masyuk AI, Huang BQ, Ward CJ, et al: Biliary exosomes influence cholangiocyte regulatory mechanisms and proliferation through interaction with primary cilia. Am J Physiol Gastrointest Liver Physiol 299: G990-G999, 2010.

28. Okaro AC, Fennell DA, Corbo M, Davidson BR and Cotter FE: Pk11195, a mitochondrial benzodiazepine receptor antagonist, reduces apoptosis threshold in $\mathrm{Bcl}-\mathrm{X}(\mathrm{L})$ and Mcl-1 expressing human cholangiocarcinoma cells. Gut 51: 556-561, 2002.

29. Lamberts RR, Van Rijen MH, Sipkema P, et al: Coronary perfusion and muscle lengthening increase cardiac contraction: different stretch-triggered mechanisms. Am J Physiol Heart Circ Physiol 283: H1515-H1522, 2002.

30. Nishino R, Honda M, Yamashita T, et al: Identification of novel candidate tumour marker genes for intrahepatic cholangiocarcinoma. J Hepatol 49: 207-216, 2008.

31. Nehls O, Gregor M and Klump B: Serum and bile markers for cholangiocarcinoma. Semin Liver Dis 24: 139-154, 2004.

32. Zhang K, Zhaos J, Liu X, et al: Activation of NF-B upregulates Snail and consequent repression of E-cadherin in cholangiocarcinoma cell invasion. Hepatogastroenterology 58: 1-7, 2011.

33. Zhang KJ, Wang DS, Zhang SY, et al: The E-cadherin repressor slug and progression of human extrahepatic hilar cholangiocarcinoma. J Exp Clin Cancer Res 29: 88, 2010.

34. Techasen A, Namwat N, Loilome W, et al: Tumor necrosis factor- $\alpha(\mathrm{TNF}-\alpha)$ stimulates the epithelial-mesenchymal transition regulator Snail in cholangiocarcinoma. Med Oncol 29: 3083-3091, 2012.

35. Zabron A, Edwards RJ and Khan SA: The challenge of cholangiocarcinoma: dissecting the molecular mechanisms of an insidious cancer. Dis Model Mech 6: 281-292, 2013.

36. Larusso NF: Patients, cells, and organelles: the intersection of science and serendipity. Hepatology 53: 1417-1426, 2011.

37. Taniai M, Grambihler A, Higuchi H, et al: Mcl-1 mediates tumor necrosis factor-related apoptosis-inducing ligand resistance in human cholangiocarcinoma cells. Cancer Res 64: 3517-3524, 2004.

38. Schwock J, Dhani N and Hedley DW: Targeting focal adhesion kinase signaling in tumor growth and metastasis. Expert Opin Ther Targets 14: 77-94, 2010.

39. Fraley SI, Feng Y, Krishnamurthy R, et al: A distinctive role for focal adhesion proteins in three-dimensional cell motility. Nat Cell Biol 12: 598-604, 2010.

40. Drukman S and Kavallaris M: Microtubule alterations and resistance to tubulin-binding agents (review). Int J Oncol 21: 621-628, 2002.

41. Pokorný J, Vedruccio C, Cifra M, Kučera O: Cancer physics: diagnostics based on damped cellular elastoelectrical vibrations in microtubules. Eur Biophys J 40: 747-759, 2011. 